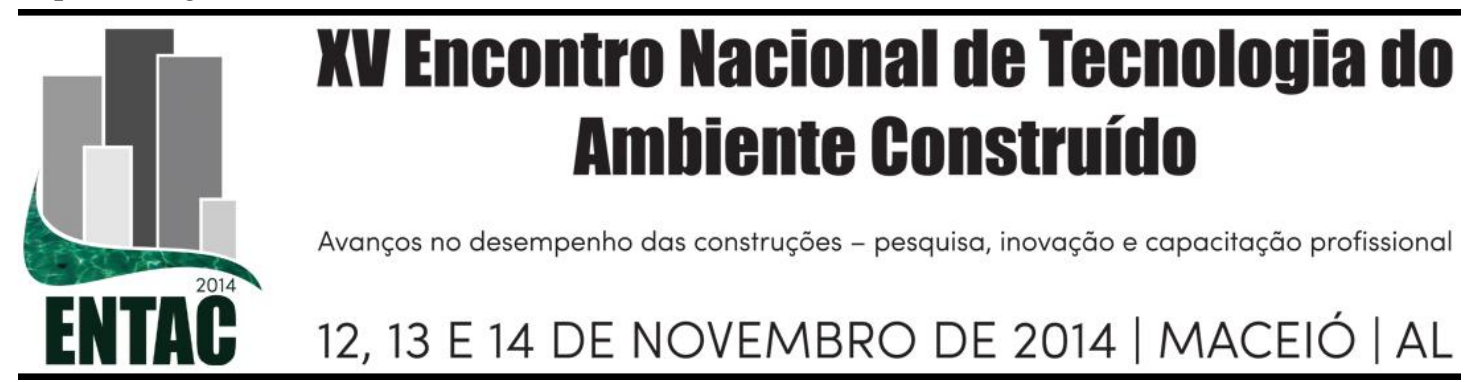

\title{
AVALIAÇÃO ESTÉTICA DE ESPAÇOS RESULTANTES ENTRE EDIFÍCIOS ALTOS
}

\author{
GREGOLETTO, Débora (1); REIS, Antônio Tarcísio da Luz (2)
}

(1) UFRGS, e-mail: deboragrego@gmail.com (2) UFRGS, e-mail: tarcisio.reis@ufrgs.br

\begin{abstract}
RESUMO
O objetivo deste artigo é avaliar a estética de espaços resultantes entre edifícios altos, considerando as percepções de grupos de usuários com diferentes níveis e tipos de formação acadêmica. Os edifícios altos estão entre os elementos construídos que mais se destacam na paisagem das cidades contemporâneas, afetando as avaliações estéticas do ambiente urbano. Edifícios altos podem afetar a estética urbana de diferentes formas: de acordo com as características da área onde estão localizados; de acordo com a distância da qual são observados; ou ainda, conforme as características dos espaços a partir dos quais tais edifícios são visualizados. Neste sentido, é importante ter uma maior compreensão sobre a qualidade estética de espaços resultantes entre edifícios altos conforme suas distintas alturas, já que estas alturas afetam a visualização da abóbada celeste, que pode ser parcialmente obstruída ou eliminada e provocar uma queda acentuada nos níveis de satisfação estética com estes espaços abertos. O fato de edifícios altos continuarem a ser construídos em grande quantidade reforça a necessidade de novas evidências sobre os impactos estéticos causados por espaços resultantes entre tais edifícios. A coleta de dados foi realizada através de questionários aplicados via internet para usuários do espaço urbano de Porto Alegre, divididos em três grupos de respondentes: 48 arquitetos; 48 não arquitetos com curso universitário completo e 33 pessoas sem curso universitário concluído ou iniciado. A análise de dados foi realizada através de testes estatísticos não-paramétricos. Os resultados indicam uma clara insatisfação com os espaços resultantes entre edifícios altos, independente do nível e tipo de formação acadêmica dos respondentes. Contudo, em contextos onde existe uma maior quantidade de céu visível, em função da presença de edifícios com menor altura, se verifica um impacto negativo menor do que em contextos onde a quantidade de céu visível é praticamente inexistente devido à maior altura dos edifícios.
\end{abstract}

Palavras-chave: avaliação estética, edifícios altos, percepção dos usuários.

\begin{abstract}
The purpose of this article is to evaluate the aesthetics of spaces between tall buildings, considering the aesthetic perceptions of different groups of users with different levels and types of academic training. Tall buildings are among the constructed elements that stand out in the landscape of contemporary cities, affecting the aesthetic evaluations of the urban environment. Tall buildings can affect urban aesthetics in different ways: according to the characteristics of urban areas where they are located; according to the distance from which they are observed; or, according to the specific features of the spaces from which such buildings are observed. In this sense, it is important to have a better understanding of the aesthetic quality of resulting spaces between tall buildings according to their different heights, since these heights affect the visualization of the celestial dome, which can be partially obstructed or eliminated and cause a sharp drop in aesthetic satisfaction levels with these open spaces. The fact that tall buildings continue to be built in large numbers, emphasizes the need for new evidence about aesthetic impacts caused by spaces resulting from such buildings. Data were collected through questionnaires distributed via the Internet to users of urban space in Porto Alegre, divided into three groups of respondents: 48 architects; 48 nonarchitects college graduates; and 33 non-college graduates. Data analysis was performed using nonparametric statistical tests. The results indicate a clear dissatisfaction with the resulting spaces between tall buildings, regardless of the level and type of academic education of the respondents. However, in situations where there is a greater amount of visible sky, due to the presence of buildings of smaller
\end{abstract}


height, it is observed a smaller negative impact than in situations where the amount of visible sky is practically nonexistent due to the greater height of buildings.

Keywords: Aesthetic evaluation, tall buildings, user's perception.

\section{INTRODUÇÃOO}

Avaliações estéticas têm sido largamente realizadas na área de estudos Ambiente e Comportamento para identificar a qualidade estética de projetos urbanos e de edificações conforme a percepção de usuários do espaço urbano. A importância destas avaliações tem sido evidenciada em diversos estudos assim como pela implementação de avaliações estéticas em cidades de diversos países como Estados Unidos, Reino Unido, Alemanha, Suécia, França, Itália e Espanha (NASAR, 1992; REIS; LAY, 2003; STAMPS, 2000; WEBER, 1995).

As avaliações estéticas são, particularmente, afetadas pela presença de edifícios altos, na medida em que estes estão entre os elementos construídos que mais se destacam na paisagem das cidades contemporâneas (DORNBUSCH; GELB, 1977; GONÇALVES, 2010; MACEDO, 1991). Os edifícios altos quando observados ao nível da linha do horizonte (do skyline de uma cidade) podem impactar positivamente na estética urbana, em razão, principalmente, da visualização à distância, além de possibilitar a consolidação da imagem das cidades enquanto potenciais econômicos e turísticos (HEAT et al., 2000; SMITH et al., 1995). Contudo, normalmente, a maioria das pessoas tem uma visão ao nível da rua (RELPH, 1987), onde vivenciam a cidade ao nível dos olhos e podem perceber os impactos das edificações e sua integração com o tecido urbano (GEHL, 2010).

Assim, fazem parte destas visuais as características dos espaços entre edifícios de múltiplos andares que constituem um componente estético importante na cidade (FORD, 2000). De acordo com o autor, os espaços entre edifícios não são apenas os espaços abertos encontrados em parques, praças e jardins, mas todos os tipos de espaços entre edifícios e imediatamente em torno deles. Estão incluídos também aqueles espaços que restaram do processo de verticalização, onde antigas edificações ou mesmo terrenos vazios ainda resistem entre os edifícios e que algumas vezes evidenciam fachadas laterais sem tratamento, que acabam afetando a estética da vizinhança (FORD, 2000).

Para Relph (1987) espaços e lugares estranhamente despojados de propriedades sensíveis e atraentes, que parecem ser o que restou depois de os edifícios terem sido erguidos, são particularmente comuns junto a edifícios altos de apartamentos. Pode haver muito espaço, mas este parece ter pouca utilidade ou ser desprovido de quaisquer formas atrativas, sendo frequentemente ajardinado de uma forma descuidada ou transformado em estacionamento. Essas áreas espaçosas raramente são locais agradáveis para uma pessoa sentar ou passear; são locais por onde se deve passar rapidamente em direção ao automóvel ou a outro edifício (RELPH, 1987). Gehl (2011) também afirma que, quando os espaços junto aos edifícios são muito grandes e impessoais, não se tornam convidativos para as atividades ao ar livre e os residentes preferem ficar em seus apartamentos. Já quando os espaços entre os edifícios são menores, com tratamento estético adequado, o trânsito de pessoas e as atividades ao ar livre se tornam mais fáceis e convidativas tendendo a um melhor funcionamento. Ainda, dependendo da altura das edificações, a visualização da abóbada celeste pode ser parcialmente obstruída ou eliminada e provocar uma queda acentuada nos níveis de satisfação estética com uma cena urbana (CIBSE, 1987; REIS et al., 2010a). Contudo, edifícios altos continuam a 
ser construídos, havendo a necessidade de novas evidências sobre os impactos estéticos causados por espaços resultantes entre edifícios altos.

Ainda, estudos (APPLEYARD; FISHMANN, 1977; SIMON, 1977; STAMPS, 1991) evidenciam a relevância da avaliação dos impactos de edifícios altos por diferentes grupos de respondentes. Por exemplo, tem sido encontradas diferenças entre as avaliações estéticas de arquitetos e de pessoas que não possuem formação na área da estética (DEVLIN; NASAR, 1989; FAWCETT et al., 2008; GIFFORD et al., 2002; SMITH et al., 1995). Por outro lado, outros estudos revelam que tal diferença quanto ao tipo de formação acadêmica não tem impacto determinante sobre as avaliações estéticas dos dois grupos (GREGOLETTO; REIS, 2012; JOHN, REIS, 2010; REIS et al., 2010). Assim, também tem sido mencionada a necessidade de haver um melhor entendimento sobre as avaliações estéticas por pessoas com diferentes níveis e tipos de formação acadêmica, principalmente na realidade brasileira (REIS et al., 2011).

Portanto, o objetivo deste trabalho é avaliar a estética de espaços resultantes entre edifícios altos, considerando as percepções estéticas de distintos grupos de usuários com diferentes níveis e tipos de formação acadêmica.

\section{METODOLOGIA}

Para atender ao objetivo proposto, o procedimento metodológico adotado foi a coleta de dados através de questionários com fotografias coloridas de espaços entre edifícios altos, aplicados via internet. Estudos (NASAR, 1992; STAMPS, 2000) corroboram a eficácia da utilização de fotografias coloridas como simuladores da realidade. A utilização da internet para divulgação e preenchimento de questionários tem sido recorrente em diversos estudos (BOCHI et al., 2012; GREGOLETTO et al., 2013; JOHN, 2012; RECKZIEGEL, 2009) e tem facilitado a obtenção de um maior número de respondentes em um curto espaço de tempo, além da redução de tempo e eliminação de possíveis erros na tabulação dos dados já que estes são transferidos diretamente do programa Lime Survey, onde os questionários estão hospedados, para a planilha do programa estatístico SPSS/PC.

O link de acesso ao questionário foi enviado via e-mail para conhecidos e solicitado a estes que reenviassem o link para os seus contatos, utilizando-se da técnica de amostra em bola de neve (snowball sample) (HANDCOCK; GILE, 2011), a fim de aumentar o número da amostra. O questionário também foi encaminhado via e-mail para empresas de engenharia e contabilidade, escritórios de arquitetura, curso pré-vestibular e secretaria da Faculdade de Arquitetura/UFRGS que repassaram o link de acesso para seus funcionários e/ou alunos. Ainda, houve a divulgação do questionário na rede social Facebook a partir da conta pessoal do primeiro autor e através de grupos específicos de arquitetos e de alunos e ex-alunos do PROPUR/UFRGS.

Os respondentes foram divididos em 3 distintos grupos, conforme o seu tipo e nível de formação acadêmica: (i) arquitetos; (ii) não arquitetos com curso universitário completo; (iii) pessoas sem curso universitário concluído ou iniciado. A amostra de respondentes dos questionários foi constituída por pessoas com idade mínima de 16 anos, residentes há pelo menos um ano em Porto Alegre. A legislação urbanística em vigor na cidade de Porto Alegre é o PDDUA (Plano Diretor de Desenvolvimento Urbano e Ambiental) e suas atualizações (PORTO ALEGRE, 2012), sendo permitidas alturas máximas de 52 metros (ou 18 pavimentos) nas principais avenidas que compõem os eixos estruturadores da malha viária; na maior parte da cidade são permitidas alturas de até 42 metros (ou 14 pavimentos). A amostra de respondentes foi constituída por 129 
respondentes, sendo 48 arquitetos, 48 não arquitetos com curso universitário completo e 33 pessoas sem curso universitário concluído ou iniciado.

O questionário estava constituído por questões fechadas de escolha simples e questões de múltipla escolha, e por questões abertas, relacionadas às três cenas representativas dos espaços entre edifícios altos (Figura 1). A Cena 1 com edifícios com 18 pavimentos foi editada para a produção das cenas com 14 e 10 pavimentos, altura esta considerada como limite para que a edificação seja considerada edifício alto, conforme identificado em outro estudo (GREGOLETTO; REIS, 2012).

\section{Figura 1 - Cenas relativas à avaliação estética dos espaços entre edifícios altos}

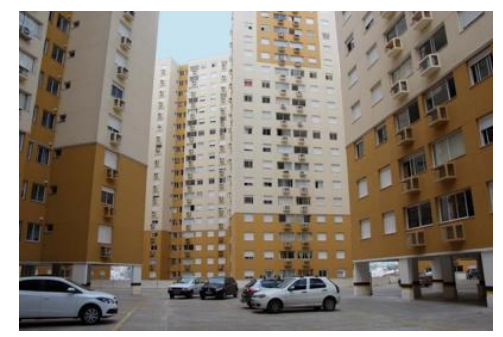

Cena 1

Original - 18 pavimentos

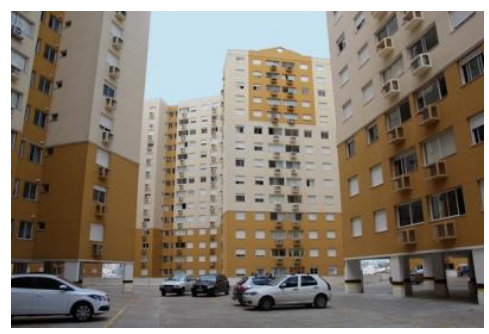

Cena 2

Simulação - 14 pavimentos

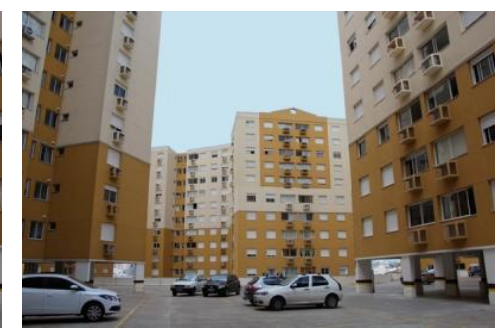

Cena 3

Simulação - 10 pavimentos

Fonte: elaborado pelo primeiro autor

Os dados de natureza quantitativa, provenientes dos questionários, caracterizados como variáveis nominais e ordinais foram analisados no programa estatístico SPSS/PC através de freqüências e de testes estatísticos não-paramétricos como: tabulação cruzada (Phi) revela relações estatisticamente significativas entre duas variáveis nominais); KruskalWallis $H$ - revela diferenças significativas entre três ou mais grupos ou amostras independentes, representados por uma variável nominal (p.ex., os três grupos de respondentes), com relação a uma variável ordinal (p.ex., avaliação estética da Cena 1) e Kendall W - revela diferenças significativas entre três ou mais grupos ou amostras dependentes, representados por uma variável nominal (p.ex., as três cenas), com relação a uma variável ordinal (avaliação estética por um dos grupos de respondentes) (LAY; REIS, 2005). Neste estudo foram consideradas as diferenças estatisticamente significativas tendo como parâmetro um valor de significância igual ou inferior a 0,05 (sig. $\leq$ 0,05) (LAY; REIS, 2005).

\section{RESULTADOS}

$\mathrm{Na}$ análise dos resultados foi encontrada uma diferença estatisticamente significativa (Kendall W, chi $^{2}=27,920$, sig. $=0,000$ ) entre a satisfação com a aparência das três cenas (Figura 1) pelo total de respondentes. Embora as três cenas tenham sido expressivamente avaliadas de forma negativa, a cena 3 (edifícios com 10 pavimentos e maior quantidade de céu visível) causou o menor impacto negativo $(63,6 \%$ de avaliações negativas e $13,2 \%$ de positivas) enquanto que a cena 1 (edifícios com 18 pavimentos) causou o maior impacto negativo $(75,2 \%$ de avaliações negativas e $8,5 \%$ de positivas) (Tabela 1 ). 


\section{Tabela 1: Satisfação com a aparência das cenas de espaços resultantes entre edifícios altos}

\begin{tabular}{|c|c|c|c|c|c|c|c|c|c|c|c|c|}
\hline \multirow{2}{*}{$\begin{array}{c}\text { Avalie a } \\
\text { aparência } \\
\text { da cena: }\end{array}$} & \multicolumn{3}{|c|}{ Total da amostra } & \multicolumn{3}{|c|}{ Arquitetos } & \multicolumn{3}{|c|}{ Não arquitetos } & \multicolumn{3}{|c|}{$\begin{array}{c}\text { Sem formação } \\
\text { universitária }\end{array}$} \\
\hline & 1 & 2 & 3 & $\begin{array}{c}\text { Cena } \\
1\end{array}$ & 2 & $\begin{array}{c}\text { Cen } \\
3\end{array}$ & 1 & 2 & \begin{tabular}{|c} 
Cena \\
3 \\
\end{tabular} & $\begin{array}{c}\text { Cena } \\
1\end{array}$ & \begin{tabular}{|c|} 
Cena \\
2 \\
\end{tabular} & $\begin{array}{c}\text { Cen } \\
3\end{array}$ \\
\hline & & 7 & o) & $\begin{array}{c}2 \\
+, 2 \%) \\
\end{array}$ & $1 \%$ & 1 & $\begin{array}{l}1 \\
2,1 \%) \\
\end{array}$ & 2 & & $\begin{array}{l}2 \\
, 1 \%) \\
\end{array}$ & $\%)$ & $\begin{array}{c}2 \\
(6,1 \% \\
\end{array}$ \\
\hline Bonita & & 2 & $\%$ & & & - & $\%)$ & $\%)$ & $\%)$ & $\%)$ & $\%)$ & $\begin{array}{c}8 \\
(24,2 \%) \\
\end{array}$ \\
\hline & $(16,3 \%)$ & 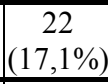 & $3,3 \%$ & \begin{tabular}{|c|}
3 \\
$(6,3 \%)$ \\
\end{tabular} & $\begin{array}{c}5 \\
(10,4 \%) \\
\end{array}$ & $\begin{array}{c}9 \\
(18,8 \%) \\
\end{array}$ & $\begin{array}{c}5 \\
(10,4 \%) \\
\end{array}$ & 6 & $\begin{array}{c}11 \\
(22,9 \%) \\
\end{array}$ & $\begin{array}{c}13 \\
(39,4 \%) \\
\end{array}$ & \begin{tabular}{|c|}
11 \\
$(33,3 \%)$ \\
\end{tabular} & $\begin{array}{c}10 \\
(30,3 \% \\
\end{array}$ \\
\hline (1a & $\begin{array}{c}39 \\
(30,2 \%) \\
\end{array}$ & 44 & 51 & $\begin{array}{c}7 \\
(14,6 \%) \\
\end{array}$ & $\begin{array}{c}9 \\
8,8 \%) \\
\end{array}$ & $\begin{array}{c}19 \\
(39,6 \%) \\
\end{array}$ & $\begin{array}{c}22 \\
(45,8 \%) \\
\end{array}$ & $\begin{array}{c}24 \\
(50,0 \%) \\
\end{array}$ & $\begin{array}{c}25 \\
(52,1 \%) \\
\end{array}$ & $\begin{array}{c}10 \\
(30,3 \%) \\
\end{array}$ & $\begin{array}{c}11 \\
(33,3 \%) \\
\end{array}$ & $\begin{array}{c}7 \\
(21,1 \%) \\
\end{array}$ \\
\hline & $\begin{array}{c}58 \\
(45,0 \%) \\
\end{array}$ & $\begin{array}{c}48 \\
(37,2 \%) \\
\end{array}$ & $\begin{array}{c}31 \\
(24,0 \%) \\
\end{array}$ & $\begin{array}{c}36 \\
(75,0 \%) \\
\end{array}$ & $\begin{array}{c}32 \\
(66,7 \%) \\
\end{array}$ & $\begin{array}{c}18 \\
(37,5 \%) \\
\end{array}$ & $\begin{array}{c}18 \\
(37,5 \%) \\
\end{array}$ & $\begin{array}{c}13 \\
(27,1 \%) \\
\end{array}$ & $\begin{array}{c}7 \\
(14,6 \%) \\
\end{array}$ & $\begin{array}{c}4 \\
(12,1 \%) \\
\end{array}$ & \begin{tabular}{|c|}
3 \\
$(9,1 \%)$ \\
\end{tabular} & $\begin{array}{c}6 \\
(18,2 \%) \\
\end{array}$ \\
\hline tal & $\begin{array}{c}129 \\
(100 \%) \\
\end{array}$ & $\begin{array}{c}129 \\
(100 \%) \\
\end{array}$ & $\begin{array}{c}129 \\
(100 \%) \\
\end{array}$ & $\begin{array}{c}48 \\
(100 \%) \\
\end{array}$ & $\begin{array}{c}48 \\
(100 \%) \\
\end{array}$ & $\begin{array}{c}48 \\
(100 \%) \\
\end{array}$ & $\begin{array}{c}48 \\
(100 \%) \\
\end{array}$ & $\begin{array}{c}48 \\
(100 \%) \\
\end{array}$ & $\begin{array}{c}48 \\
(100 \%) \\
\end{array}$ & $\begin{array}{c}33 \\
(100 \%) \\
\end{array}$ & $\begin{array}{c}33 \\
(100 \%) \\
\end{array}$ & $\begin{array}{c}33 \\
(100 \%) \\
\end{array}$ \\
\hline & 2,20 & 2,03 & 1,77 & 2,24 & & 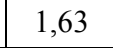 & 2,24 & & 1,77 & 2,08 & 5 & ,97 \\
\hline nvo K-W & - & - & - & 84,46 & 84,57 & 78,00 & 64,23 & 62,52 & 63,19 & 37,82 & 40,14 & 48,73 \\
\hline
\end{tabular}

Notas: mvo K= média dos valores ordinais obtida pelo teste Kendall's W (os valores menores referem-se às cenas mais satisfatórias); a comparação entre os valores mvo $\mathrm{K}$ deve ser feita na horizontal entre as cenas de cada grupo; mvo K-W= média dos valores ordinais obtida pelo teste Kruscal-Wallis (os valores menores referem-se aos grupos mais satisfeitos); a comparação entre os valores mvo K deve ser feita na horizontal entre as cenas de cada grupo; a comparação entre os valores mvo K-W deve ser feita na horizontal entre os grupos e as cenas; os valores entre parênteses referem-se aos percentuais em relação ao total de respondentes que avaliou cada cena em cada grupo.

Fonte: elaborado pelos autores

Tabela 2: Preferência em cenas de espaços resultantes entre edifícios altos

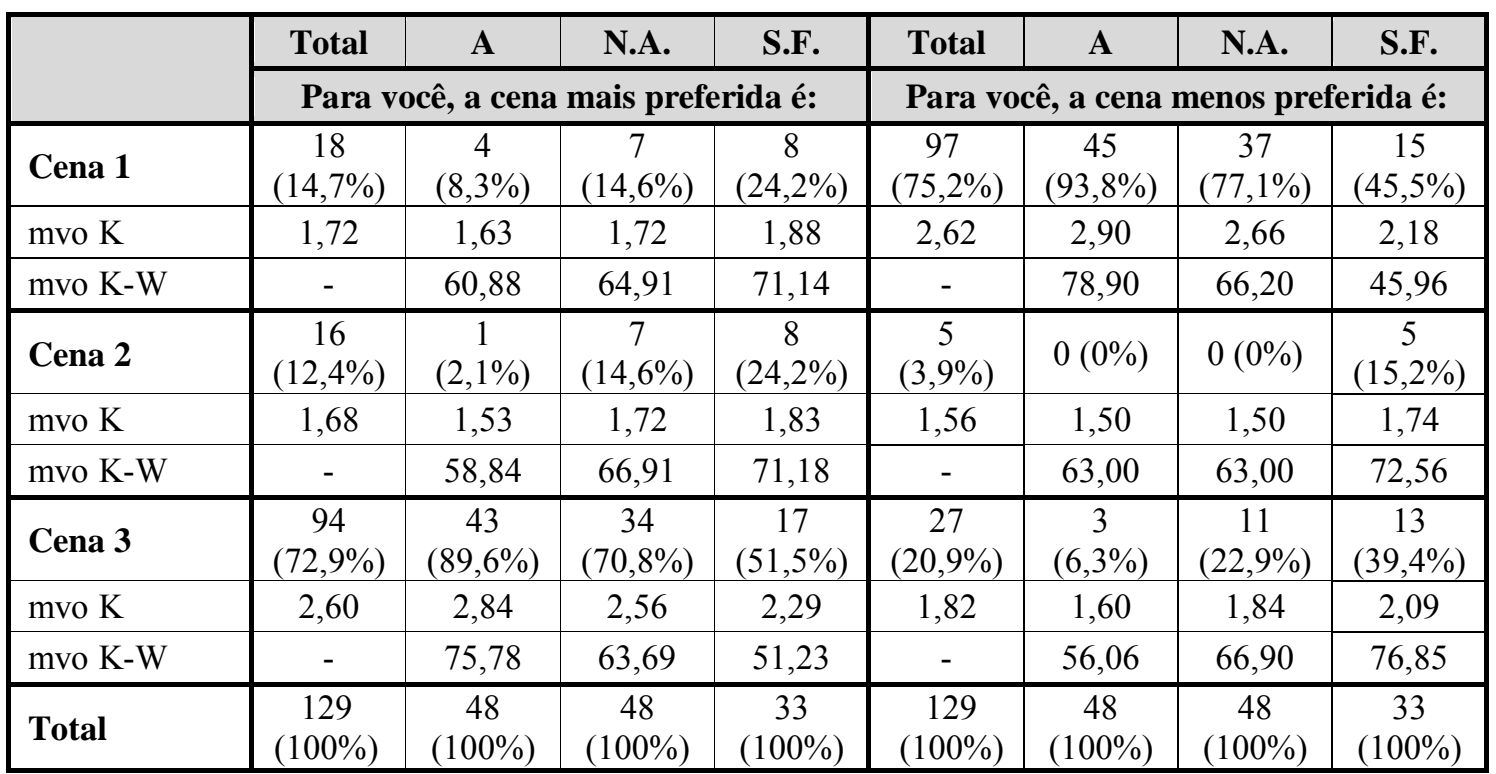

Notas: $A=$ Arquitetos; N.A.= Não arquitetos; S.F..= Respondentes sem formação universitária; mvo $\mathrm{K}=$ média dos valores ordinais obtida pelo teste Kendall's W (os valores maiores referem-se às cenas mais preferidas); mvo $\mathrm{K}-\mathrm{W}=$ média dos valores ordinais obtida pelo teste Kruscal-Wallis (os valores maiores referem-se aos grupos que mais preferem a cena); a comparação entre os valores mvo K deve ser feita na vertical entre as cenas de cada grupo; os valores entre parênteses referem-se aos percentuais em relação ao total de respondentes que avaliou cada cena em cada grupo.

Fonte: elaborado pelos autores 
Também foi encontrada uma diferença estatisticamente significativa entre as preferências do total dos respondentes quanto à cena mais preferida (Kendall W, $\mathrm{chi}^{2}=92,828$, sig. $=0,000$ ) e à cena menos preferida (Kendall W, chi $^{2}=105,672$, sig. $=0,000)$ pelo total da amostra (Tabela 2 ).

A cena 3 (edifícios com 10 pavimentos) foi a mais preferida pela maioria dos respondentes (Tabela 2), em razão, fundamentalmente, da "quantidade adequada de céu visível" e da "altura adequada das edificações" (Tabela 3). A cena 1 (edifícios com 18 pavimentos) foi considerada a menos preferida pela maioria dos respondentes (Tabela 2), devido, principalmente, à "altura inadequada das edificações" e à "quantidade inadequada de céu visível" (Tabela 3 ).

Tabela 3: Principais razões que justificam a preferência em cenas de espaços resultantes entre edifícios altos

\begin{tabular}{|c|c|c|c|c|}
\hline \multicolumn{5}{|c|}{ Indique as principais razões que justifiquem a cena mais preferida: } \\
\hline Razões & Cena 1 & Cena 2 & Cena 3 & Total \\
\hline Altura adequada das edificações & $13(10,1 \%)$ & $12(9,3 \%)$ & $31(24,0 \%)$ & $56(43,4 \%)$ \\
\hline $\begin{array}{l}\text { Quantidade adequada de céu } \\
\text { visível }\end{array}$ & $4(3,1 \%)$ & $4(3,1 \%)$ & $52(40,3 \%)$ & $60(46,5 \%)$ \\
\hline $\begin{array}{l}\text { Proporção mais adequada entre } \\
\text { a altura e a distancia dos } \\
\text { edifícios }\end{array}$ & $0(0 \%)$ & $0(0 \%)$ & $3(2,3 \%)$ & $3(2,3 \%)$ \\
\hline Maior visibilidade & $0(0 \%)$ & $2(1,6 \%)$ & $2(1,6 \%)$ & $4(3,1 \%)$ \\
\hline \multicolumn{5}{|c|}{ Indique as principais razões que justifiquem a cena menos preferida: } \\
\hline Razões & Cena 1 & Cena 2 & Cena 3 & Total \\
\hline $\begin{array}{l}\text { Altura inadequada } \\
\text { edificações }\end{array}$ & $64(49,6 \%)$ & $1(0,8)$ & $19(14,7)$ & $84(65,1 \%)$ \\
\hline $\begin{array}{l}\text { Quantidade inadequada de céu } \\
\text { visível }\end{array}$ & $49(38,0 \%)$ & $4(3,1)$ & $6(4,7)$ & $59(45,7 \%)$ \\
\hline $\begin{array}{l}\text { Pouco afastamento entre } \\
\text { edifícios }\end{array}$ & $7(5,4 \%)$ & $0(0 \%)$ & $0(0 \%)$ & $7(5,4 \%)$ \\
\hline Ambiente inóspito, árido & $4(3,1 \%)$ & $0(0 \%)$ & $0(0 \%)$ & $4(3,1 \%)$ \\
\hline Ausência de vegetação & $3(2,3 \%)$ & $0(0 \%)$ & $0(0 \%)$ & $3(2,3 \%)$ \\
\hline Falta de proporção & $0(0 \%)$ & $0(0 \%)$ & $3(2,3 \%)$ & $3(2,3 \%)$ \\
\hline Estética das edificações & $3(2,3 \%)$ & $0(0 \%)$ & $0(0 \%)$ & $3(2,3 \%)$ \\
\hline Sensação de opressão & $2(1,6 \%)$ & $0(0 \%)$ & $0(0 \%)$ & $2(1,6 \%)$ \\
\hline Sensação de insegurança & $1(0,8 \%)$ & $0(0 \%)$ & $0(0 \%)$ & $1(0,8 \%)$ \\
\hline
\end{tabular}

Nota: os valores entre parênteses referem-se aos percentuais em relação ao total de respondentes Fonte: elaborado pelos autores

Especificamente, também foi encontrada uma diferença estatisticamente significativa (Kendall W, chi $^{2}=26,237$, sig. $=0,000$ ) quanto à satisfação com a aparência das três cenas pelos arquitetos. Embora as 3 cenas tenham causado claro impacto estético negativo, o menor impacto foi verificado na cena 3 (edifícios com 10 pavimentos) com $77,1 \%$ de avaliações negativas e $4,2 \%$ de positivas, enquanto que na cena 1 (edifícios com 18 pavimentos) foi verificado maior impacto negativo com $89,6 \%$ de avaliações negativas e 4,2\% de avaliações positivas (Tabela 1). Ainda, foi encontrada uma diferença estatisticamente significativa em relação à cena mais preferida (Kendall W, chi $^{2}=68,625$, sig. $\left.=0,000\right)$ e menos preferida $\left(\right.$ Kendall W, chi $^{2}=77,149$, sig. $\left.=0,000\right)$ pelos arquitetos, confirmando a cena 3 (edifícios com 10 pavimentos) como a mais preferida e a cena 1 (edifícios com 18 pavimentos) como a menos preferida (Tabela 2). 
Adicionalmente, foi encontrada uma diferença estatisticamente significativa (Kendall $\mathrm{W}, \mathrm{chi}^{2}=13,703$, sig. $\left.=0,001\right)$ quanto à satisfação com a aparência das três cenas pelos não arquitetos com curso universitário. A cena 3 (edifícios com 10 pavimentos) causou o menor impacto negativo com $66,7 \%$ de avaliações negativas e $10,4 \%$ de positivas, enquanto que a cena 1 (edifícios com 18 pavimentos) causou o maior impacto negativo com $83,3 \%$ de avaliações negativas e $6,3 \%$ de avaliações positivas (Tabela 1). Ainda foi encontrada uma diferença estatisticamente significativa relação à cena mais preferida (Kendall W, chi $^{2}=30,375$, sig. $=0,000$ ) e menos preferida (Kendall W, chi ${ }^{2}=45,125$, sig. $=0,000)$ pelos não arquitetos com curso universitário, confirmando a cena 3 (edifícios com 10 pavimentos) como a mais preferida e a cena 1 (edifícios com 18 pavimentos) como a menos preferida (Tabela 2).

Por outro lado, não foram encontradas diferenças estatisticamente significativas quanto à satisfação com a aparência das três cenas pelas pessoas sem formação universitária, visto que as três cenas tiveram avaliação semelhante. Também não foram encontradas diferenças estatisticamente significativas em relação à cena mais preferida e à cena menos preferida por este grupo de respondentes.

Os resultados revelam uma clara insatisfação com a aparência das cenas que representam espaços resultantes entre edifícios altos, tanto para o total da amostra, quanto para os três grupos de respondentes. Porém, as cenas que apresentam edificações mais baixas e consequente maior quantidade de céu visível, tendem a causar um impacto negativo menor do que as cenas onde a quantidade de céu visível é praticamente inexistente.

\subsection{Diferença entre os grupos de usuários}

A existência de diferenças quanto à satisfação com a aparência de uma mesma cena por pessoas com diferentes níveis e tipos de formação, foi analisada através do teste estatístico Kruskal-Wallis. Foi encontrada uma diferença estatisticamente significativa entre arquitetos, não arquitetos com curso universitário e pessoas sem formação universitária quanto à satisfação com a aparência da cena 1 (edifícios com 18 pavimentos) $\left(\mathrm{K}-\mathrm{W}, \mathrm{chi}^{2}=34,744\right.$, sig. $\left.=0,000\right)$, da cena 2 (edifícios com 14 pavimentos) $\left(\mathrm{K}-\mathrm{W}\right.$, chi $^{2}=30,955$, sig. $\left.=0,000\right)$ e da cena 3 (edifícios com 10 pavimentos) (K-W, $\mathrm{chi}^{2}=13,367$, sig. $=0,001$ ). Embora tenha predominado a avaliação negativa nas três cenas pelos três grupos de respondentes, um maior impacto estético negativo parece ter sido percebido pelos arquitetos e não arquitetos com curso universitário do que pelas pessoas sem formação universitária (Tabela 1).

Ainda, foi encontrada uma diferença estatisticamente significativa entre arquitetos, não arquitetos com curso universitário e pessoas sem formação universitária, quanto à maior preferência pela cena 2 (edifícios com 14 pavimentos) $\left(\mathrm{K}-\mathrm{W}\right.$, chi $^{2}=7,554$, sig. $\left.=0,023\right)$, já que a cena foi um pouco mais citada como a mais preferida pelas pessoas sem formação universitária do que pelos demais respondentes (Tabela 2). Também foi encontrada uma diferença estatisticamente significativa entre os três grupos de respondentes quanto à maior preferência pela cena 3 (edifícios com 10 pavimentos) (K-W, chi² $=14,382$, sig. $=0,001$, pois ainda que a cena tenha sido considerada a mais preferida pelos três grupos de respondentes, a cena foi mais preferida pelos arquitetos e pelos não arquitetos do que pelas pessoas em formação universitária (Tabela 2).

Também foi encontrada uma diferença estatisticamente significativa entre os três grupos de respondentes quanto à menor preferência pela cena 1 (K-W, chi ${ }^{2}=25,543$, sig. $=0,000$ ). Embora a cena tenha sido considerada a menos preferida pelos três grupos 
de respondentes, a cena foi claramente mais citada como a menos preferida pelos arquitetos e pelos não arquitetos com formação universitária do que pelas pessoas sem formação universitária (Tabela 2). Ainda, foi encontrada uma diferença estatisticamente significativa entre arquitetos, não arquitetos com curso universitário e pessoas sem formação universitária, quanto à menor preferência pela cena $2\left(\mathrm{~K}-\mathrm{W}\right.$, chi $^{2}=14,569$, sig. $=0,001)$ e pela cena $3\left(\mathrm{~K}-\mathrm{W}, \mathrm{chi}^{2}=12,490\right.$, sig. $\left.=0,002\right)$, onde as pessoas sem formação universitária indicaram essas duas cenas como a menos preferida com uma maior intensidade do que os demais grupos de respondentes (Tabela 2).

Portanto, essas diferenças evidenciam a avaliação negativa das cenas que representam espaços entre edifícios altos, independente do tipo e da formação acadêmica dos respondentes, embora possam ocorrer variações nas intensidades das avaliações e preferências.

\section{CONSIDERAÇÕES FINAIS}

Os resultados da avaliação estética revelaram uma clara insatisfação com a aparência dos espaços resultantes entre edifícios altos, tanto para o total da amostra, quanto para os três grupos de respondentes. Contudo, no contexto onde existe uma maior quantidade de céu visível em função de edifícios com menor altura (10 pavimentos), se verifica um impacto negativo menor do que nos contextos onde a quantidade de céu visível é praticamente inexistente devido à maior altura dos edifícios (18 pavimentos). Assim, a maior altura das edificações contribuiu com a insatisfação com a aparência dos espaços resultantes entre edifícios altos em função da diminuição da quantidade de céu visível. Logo, estes resultados ratificam aqueles de outros estudos (CIBSE, 1987; REIS et al., 2010a) que revelam queda acentuada nos níveis de satisfação estética quando a visualização da abóboda celeste é parcialmente obstruída ou eliminada de uma cena urbana. Além das alturas das edificações, o impacto estético negativo também parece estar relacionado às características físicas desses espaços, despojados de propriedades atraentes e sem tratamento estético adequado, tanto no nível do solo quando nos edifícios, tornando-se locais por onde se deve passar rapidamente em direção ao automóvel ou a outro edifício (FORD, 2000; GEHL, 2010; RELPH, 1987). Portanto, a ocupação das cidades por edifícios altos também facilita o surgimento de espaços impessoais, residuais, sem identidade, uso e qualidade estética. Concluindo, espaços entre edifícios mais baixos (p.ex., com 10 pavimentos), com uma maior visualização da abóbada celeste, e com tratamento estético adequado, se tornariam mais atraentes e potencializariam o fluxo de pessoas e a realização de atividades ao ar livre, impactando positivamente os usuários de tais edifícios. Assim, espera-se que os resultados obtidos possam contribuir para uma maior compreensão acerca da estética de espaços resultantes entre edifícios altos e possam retroalimentar futuros projetos de edifícios altos através da criação de bancos de dados baseados nas percepções dos usuários do espaço urbano, tanto da cidade de Porto Alegre quanto de outras cidades, uma vez que a metodologia adotada pode ser reaplicada em diferentes contextos. Ainda, deseja-se que as conclusões desse estudo possam contribuir para o planejamento urbano na discussão e elaboração de legislações urbanísticas que regulamentam as alturas dos edifícios nas cidades, para uma melhor adequação das alturas dos edifícios e espaços abertos resultantes de maneira a melhor qualificar esteticamente as cidades. 


\section{REFERÊNCIAS}

APPLEYARD, D.; FISHMAN, L. High rise buildings versus San Francisco: Measuring visual and symbolic impacts. In: CONWAY, D. (Ed.). Human response to tall buildings. Stroudsburg: Dowden, Hutchinson \& Ross, 1977. P. 81-100.

BOCHI, T. C.; GREGOLETTO, D.; REIS; A. T. L. Cercamento de parques urbanos conforme a percepção de usuários comerciantes. In: XXXI Encuentro y XVI Congreso Arquisur, 2012, Buenos Aires. Anais... Buenos Aires: ARQUISUR, 2012.

CHARTERED INSTITUTION OF BUILDING SERVICES ENGINEERS, THE (CIBSE). Applications manual: window design. London: The Chartered Institution of Building Services Engineers, 1987.

DEVLIN, K. ; NASAR, J. L. The beauty and the beast: Some preliminary comparisons of "high" versus "popular" residential architecture and public versus architect judgments of same. Journal of Environmental Psychology, v. 9, n. 4, p. 333-344, 1989.

DORNBUSCH, D.; GELB, P. High rise visual impact. In: CONWAY, D. (Ed.). Human response to tall buildings. Stroudsburg: Dowden, Hutchinson \& Ross, 1977. p. 101-111.

FAWCETT, W. ; ELLINGHAM, I. ; PLATT, S. Reconciling the Architectural Preferences of Architects and the Public: The Ordered Preference Model. Environment and Behavior, v. 40, n. 5, p. 599-618, 2008.

FORD, L. R. The Spaces between Buildings. Baltimore: The Johns Hopkins University Press, 2000.

GEHL. J. Cities for people. Washington: Island Press, 2010.

GEHL, J. Life between buildings: using public space. Washington: Island Press, 2011.

GIFFORD, R.; HINE, D. W.; MULLER-CLEMM, W.; SHAW, K. T. Why architects and laypersons judge buildings differently: cognitive properties and physical bases. Journal of Architecture and Planning Research, v. 19, n. 2, p. 131-148, 2002.

GONÇALVES, J.C.S. The environmental performance of tall buildings. London: Earthscan, 2010.

GREGOLETTO, D.; REIS, A. T. L. Os edifícios altos na percepção dos usuários do espaço urbano. Cadernos Proarq, n. 19, 2012. P. 90-110.

GREGOLETTO, D.; BOCHI, T. C.; SILVA, F. C.; REIS, A. T. L. Existência e inexistência de cercamento, segurança e acessibilidade de parques urbanos. Arquisur Revista, ano 3, n.3, 2013. P. 124-137.

HANDCOCK, M.; GILE, K. On the Concept of Snowball Sampling. Sociological Methodology, v. 41, P. 367-371, 2011.

HEAT, T; SMITH, S. G.; LIM, B. Tall Buildings and the Urban Skyline : The Effect of Visual Complexity on Preferences. Environment and Behavior, v. 32, n. 4, P. 541-556, 2000.

JOHN, N. M.; REIS, A. T. L. Mobiliário urbano e paisagem urbana: avaliação estética de abrigos de transporte coletivo. In: ENCONRO NACIONAL DE TECNOLOGIA DO AMBIENTE CONSTRUIIDO, XIII, Canela, 2010. Anais... Canela: ANTAC, 2010.

JOHN, N. M. Avaliação estética do mobiliário urbano e do uso de abrigos de ônibus por cadeirantes. Porto Alegre: UFRGS, 2012. Dissertação (Mestrado em Planejamento Urbano e Regional). Faculdade de Arquitetura, Universidade Federal do Rio Grande do Sul, Porto Alegre, 2012.

LAY, M. C.; REIS, A. T. L. Análise quantitativa na área de estudos ambiente-comportamento. Revista Ambiente Construído, Porto Alegre, v.5, n.2, p.21-36, 2005. 
MACEDO, S. S. O processo de verticalização e a paisagem da cidade. Sinopses, São Paulo, v. 15, p.68-76, 1991.

NASAR, J. L. Environmental Aesthetics: theory, research, and applications. New York: Cambridge University Press, 1992.

PORTO ALEGRE. Lei Complementar 434/99 atualizada e compilada até a Lei Complementar 677/11, incluindo a Lei Complementar 646/10. Plano Diretor de Desenvolvimento Urbano e Ambiental (PDDUA). Porto Alegre, 2012.

RECKZIEGEL, D. Lazer noturno: aspectos configuracionais e formais e sua relação com a satisfação e preferência dos usuários. Porto Alegre: UFRGS, 2009. Dissertação (Mestrado em Planejamento Urbano e Regional). Faculdade de Arquitetura, Universidade Federal do Rio Grande do Sul, Porto Alegre, 2009.

REIS, A. T. L.; LAY, M. C. "Habitação de interesse social: uma análise estética". Revista Ambiente Construído, Porto Alegre, v. 3, n. 4, p. 7-19, 2003.

REIS, A. T. L.; BIAVATTI, C.; PEREIRA, M. L. Avaliação estética de cenas urbanas históricas e contemporâneas com diferentes níveis de ordem e estímulo visual. In: ENCONTRO NACIONAL DE TECNOLOGIA DO AMBIENTE CONSTRUÍDO, XIII, 2010, Canela. Anais... Canela: ANTAC, 2010.

REIS, A. T. L.; PEREIRA, M. L.; BIAVATTI, C. Percepção visual e impacto estético de vistas a partir de apartamentos. In: ENCONTRO NACIONAL DE TECNOLOGIA DO AMBIENTE CONSTRUÍDO, XIII, 2010, Canela. Anais... Canela: ANTAC, 2010a.

REIS, A; BIAVATTI, C.; PEREIRA, M. L. Estética urbana: uma análise através das ideias de ordem, estímulo visual, valor histórico e familiaridade. Revista Ambiente Construído, Porto Alegre, v. 11, n. 4, p. 185-204, out./dez. 2011.

RELPH, E. Paisagem urbana moderna. Lisboa: Edições 70, 1987.

SIMON, L. The impact of high-rise structures on the community. In: CONWAY, D. (Ed.) Human response to tall buildings. Stroudsburg: Dowden, Hutchinson \& Ross, 1977. P. 41-44.

SMITH, S. G.; HEATH, T.; LIM, B. The influence of building height and spacing on the evaluation of city skylines: A comparison between architects and non-architects. In: ANNUAL CONFERENCE OF THE ENVIRONMENTAL DESIGN RESEARCH ASSOCIATION, 26, 1995, Boston. Anais... Boston: EDRA 26, 1995. P. 65-69.

STAMPS, A. E. Public preference for high rise buildings: Stylistic and demographic effects. Perceptual and Motor Skills, 72, p. 839-844, 1991.

STAMPS A. E. Psychology and the Aesthetics of the Built Environment. Norwell: Kluwer Academic Publishers, 2000.

WEBER, R. On the Aesthetics of architecture: a Psychological Approach to the Structure and the Order of Perceived Architectural Space. Aldershot, UK: Avebury, 1995. 